\title{
Yes-associated protein gene overexpression regulated by $\beta$-catenin promotes gastric cancer cell tumorigenesis
}

\author{
Tianzhu Qiu ${ }^{\mathrm{a}, 1}$, Diancai Zhang ${ }^{\mathrm{b}, 1}$, Jing Xu ${ }^{\mathrm{a}, 1}$, Xiao Li ${ }^{\mathrm{c}}$, Deqiang Wang ${ }^{\mathrm{d}}$, Fengjiao Zhao ${ }^{\mathrm{a}}$, \\ Yingying Qian ${ }^{\mathrm{e}}$, Jin $\mathrm{Xu}^{\mathrm{f}}$, Tongpeng $\mathrm{Xu}^{\mathrm{a}}$, Hao Zhang ${ }^{\mathrm{a}, *}$ and Xiaofeng Chen ${ }^{\mathrm{a}, \mathrm{g}, *}$ \\ ${ }^{a}$ Department of Oncology, First Affiliated Hospital of Nanjing Medical University, Nanjing, Jiangsu, \\ China \\ ${ }^{\mathrm{b}}$ Department of General Surgery, First Affiliated Hospital of Nanjing Medical University, Nanjing, \\ Jiangsu, China \\ ${ }^{\mathrm{c}}$ Department of Pathology, First Affiliated Hospital of Nanjing Medical University, Nanjing, Jiangsu, \\ China \\ ${ }^{\mathrm{d}}$ Department of Medical Oncology, Affiliated Hospital of Jiangsu University, Zhenjiang, Jiangsu, China \\ ${ }^{\mathrm{e}}$ Department of Respiratory, Nanjing First Hospital, Nanjing Medical University Nanjing, Jiangsu, China \\ ${ }^{\mathrm{f}}$ Department of Maternal, Child and Adolescent Health, School of Public Health, Nanjing Medical \\ University, Nanjing, China \\ ${ }^{g}$ Department of Oncology, PuKou Branch Hospital of Jiangsu Province Hospital (NanJing PuKou \\ Central Hospital), Nanjing, Jiangsu, China
}

\begin{abstract}
.
BACKGROUND: Yes-associated protein (YAP) has been reported to act as a candidate human oncogene and played a critical role in the development of multiple cancer types.

OBJECTIVE: We aimed to investigate the expression, function, and underlying mechanisms of YAP in gastric cancer (GC). METHODS: Expression levels of YAP in gastric tissues were tested. CCK8 assay, clonogenic assay, apoptosis assay, transwell assay, cell scratch assay and animal study were conducted to explore the function of YAP. Chromatin immunoprecipitation (ChIP) assay and luciferase reporter assay were performed to explore the underlying mechanism. Survival analysis was carried out to reveal the relationship between YAP and clinical outcome.

RESULTS: YAP was upregulated in gastric cancer tissues and correlates with poor prognosis. YAP could promote GC cells proliferation, metastatic capacity, inhibit GC cells apoptosis in vitro and in vivo. Both $\beta$-catenin and YAP were mainly localized withi the tumor cell nuclei. $\beta$-catenincould upregulate YAP expression by binding to the promotor region of YAP. Patients with both YAP and $\beta$-catenin negetive expression had a better prognosis than others.

CONCLUSIONS: YAP overexpression is driven by aberrant Wnt $\beta$-catenin signalingand then contributed to the GC tumorigenesis and progression. Thus, YAP might be a potential target for GC treatment.

Keywords: YAP, $\beta$-catenin, gastric cancer, prognosis, tumorigenesis

\footnotetext{
${ }^{1}$ These authors contributed equally to this work.

${ }^{*}$ Corresponding authors: Xiaofeng Chen, Department of Oncology, First Affiliated Hospital of Nanjing Medical University, 300 Guangzhou Road, Nanjing, Jiangsu 210029, China. Tel.: 13585172066; E-mail: chenxiaofengnjmu@ 163.com. Hao Zhang, Department of Oncology, First Affiliated Hospital of Nanjing Medical University, 300 Guangzhou Road, Nanjing, Jiangsu 210029, China. Tel.: 13851798920; E-mail: dndxzh@njmu.edu.cn
}

0928-7329 (c) 2022 - The authors. Published by IOS Press. This is an Open Access article distributed under the terms of the Creative Commons Attribution-NonCommercial License (CC BY-NC 4.0). 


\section{Introduction}

Gastric cancer (GC) is the fourth most common cancer and the second highest cause of cancer-related mortality in the world [1]. Despite many years of significant advances in early detection and treatment, the majority of patients with GC are diagnosed at an advanced, unresectable stage, resulting in poor prognosis [2]. Thus it is highly desirable to establish new therapeutic target and more effective strategy in gastric cancer therapy.

Hippo signaling pathway is highly conserved and regulates tissue stability, controls organ size and governs stem cell self-renewal [3-5]. Hippo signaling pathway's major downstream target is Yesassociated protein (YAP). YAP promotes cell proliferation, inhibits cellular apoptosis and suppresses cancer immunity [6-9]. YAP has recently been identified as a potential human oncogene and plays a key role in the genesis and tumorigenesis, including gastric cancer [10-12]. However, the relationship between YAP, as well as Hippo pathway, and the prognosis of GC patients have not been thoroughly investigated.

Although it appears that direct mutations in the Hippo pathway are infrequent, multiple cancer-related signaling networks interact with the Hippo pathway in regulatory crosstalk, generally at YAP onco-protein level $[13,14]$. Yap is hyper-activated in multiple cancers [15]. Wnt $\beta$-catenin pathway is essential in cell proliferation, differentiation, embryo development and tissue homeostasis [16,17]. Gene mutations of this pathway causing hyper-activation are linked to the development of numerous cancers. Growing evidence suggests that Wnt/-catenin pathways and Hippo pathways interact in a variety of ways to regulate tissue growth and repair $[18,19]$. However, the underlying crosstalk mechanisms between the two pathways in GC are still not clear

In this study, we investigated the expression of YAP in gastric cancer and the role YAP played in proliferation, apoptosis, migration and invasion. Furthermore, we found $\beta$-catenin participates in Yap transcription. High YAP expression in GC tissues was associated with a poorer prognosis. Therefore, YAP may serve as a potential target in GC therapy.

\section{Material and methods}

\subsection{Clinical samples}

The study complied with the Declaration of Helsinki and was approved by the Ethics Committees of the First Affiliated Hospital of Nanjing Medical University. Legally effective informed consent was gained from all patients. Training set contains $28 \mathrm{GC}$ samples and adjacent normal tissues collected from the First Affiliated Hospital of Nanjing Medical University between July 2010 and September 2013, while validation set contains 101 gastric cancer samples from the First Affiliated Hospital of Nanjing Medical University between July 2007 and August 2012. None of these patients received neoadjuvant therapy prior to surgery.

\subsection{Immunohistochemistry (IHC)}

Embedded tumor tissues were sliced to $4 \mathrm{~mm}$ thick, and then dewaxed. After heat-induced antigen retrieval in citrate buffer, $3 \%$ hydrogen peroxide was used. Slices were then incubated at $4^{\circ} \mathrm{C}$ with the appropriate YAP1 antibodies (ab52771, Abcam) overnight. IHC Kit was used to further incubate the sections, which were then stained with 3,30-diamino-benzidine tetrahydrochloride and hematoxylin. 


\subsection{Cell culture and transfection}

Gastric cancer cell lines that including SGC-7901, MGC803, BGC-823 used in our study were obtained from China Center Type Culture Collection (CCTCC, Shanghai, China). Invasion ability varied from high to low as such sequence: MGC-803, BGC-823 to SGC-7901. BGC-823 was cultured in RPMI 1640 while SGC-7901 and MGC803 cell lines were cultured in DMEM, supplementing with heat-inactivated FBS. Plasmids encoding YAP shRNA were purchased from Guangzhou RiboBio (RiboBio, Guangzhou, China). Plasmid transfection was performed with Lipofectamine 2000 transfection reagent (Invitrogen). Briefly, gastric cancer cells were seeded in $60-\mathrm{mm}$ dishes to be at $50-70 \%$ confluence on the day of transfection and the medium was changed $24 \mathrm{~h}$ post-transfection. Plasmids and Lipofectamine 2000 were mixed and incubated for $5 \mathrm{~min}$. Complexes were added together and incubated for $20 \mathrm{~min}$. Silencing of proteins was monitored for $72 \mathrm{~h}$ after transfection by western blot analysis.

\section{4. $q R T-P C R$}

Total RNA was isolated using the TRIzol reagent (Invitrogen). To obtain cDNAs, M-MLV reverse transcriptase (Promega) and OligodT primers (Sangon, Shanghai) were used for reverse transcription. The primers for YAP forward were 5'-GCA ACT CCA ACC AGC AGC AACA-3' and for YAP reverse were 5'-CGC AGC CTC TCC TTC TCC ATCTG-3'. All samples were examined in triplicates.

\subsection{Western blot}

Cells were seeded in and cultured in 6-well plates before Western blot. Then we extracted total proteins and separated them on $10 \%$ sodium dodecyl sulfate-polyacrylamide gels. After that, we conducted western blot as described previously [20], using goat anti-mouse IgG (Santa Cruz Biotechnology).

\subsection{Subcellular fractionation}

We transferred cells from plates into fractionation buffer then lysed all cells by passing cell suspension with needle repeatedly. Then we leaved cell suspension on ice for $20 \mathrm{~min}$. After that, we centrifuged samples, transferred the supernatant and kept them on ice and washed nuclear pellet and disperse the pellet. After centrifuging the pellet again, supernatant was discarded, while the pellet containing nuclei was retained. Then we resuspended the pellet and centrifuge the supernatant.

\subsection{CCK8 assay}

We use Cell Counting Kit-8 (CCK8) detection kit (KeyGEN, Jiangsu, China) to measure SGC-7901 and BGC-823 cells proliferation. After transfection, cells were planted in 96-well plates at a concentration of 2000 cells per well. CCK-8 solution was applied to each well and incubated at $37^{\circ} \mathrm{C}$ at $24 \mathrm{~h}, 48 \mathrm{~h}, 72 \mathrm{~h}$ and $96 \mathrm{~h}$ after seeding. Then we measure absorbance value at $450 \mathrm{~nm}$ spectrophotometrically.

\subsection{Clonogenic assay}

Three days after transfection, BGC-823 and SGC-7901 cells were cultured and collected. After that, cells were plated into 6-well plates ( 800 cells/well). Cells were washed and fixed for 60 min after being incubated in a $5 \% \mathrm{CO}_{2}$ incubator at $37^{\circ} \mathrm{C}$ for 11 days. After staining with Giemsa (ECM550, chemicon), we counted the number of clones and calculated the relative percentage. 


\subsection{Apoptosis assay}

Apoptosis was evaluated by Flow cytometry analyses and fluorescence-activated cell sorting (FACS), using the Annexin V-APC staining method (Apoptosis Detection Kit, MBL, Japan). Briefly, after trypsinization, cells were resuspended in a centrifuge tube. Unbound supernatant was then removed by a centrifugal wash and stained by Annexin V-APC.

\subsection{Transwell assay}

BGC823 and SGC7901 cells were plated onto 24-well MilliCell chambers (Millipore, Bedford, MA, USA) at a count of $1 * 10^{5}$. Then we seeded them in media containing $0.1 \%$ serum and put chambers into 24-well plates in $10 \%$ serum media. Invaded cells were fixed and stained with 0.1 percent crystal violet on the lower membrane surface after 24 hours.

\subsection{Cell scratch assay}

A scratch wound experiment was used to test the ability of cells to disseminate and migrate. After transfection with YAP shRNA, we plated BGC823 and SGC7901 cells into 12-well tissue culture dishes which were coated coverslips. Then we draw a linear wound. Twenty-four hours later, we observed cells with microscope and measured them with software.

\subsection{Chromatin immunoprecipitation (ChIP) assay}

ChIP assays were performed according to the product instructions using the ChIP Assay Kit (P2078, Beyotime, China). Briefly, the samples were sonicated and immunoprecipitated in rotating overnight at $4^{\circ} \mathrm{C}$. The recovered DNA samples were utilized as templates for PCR using the ABI Prism 7500 System with Tli RNaseH Plus kit. Then the degree of enrichment was determined by qRT-PCR. TCF4 antibody was purchased from Abcam. The primers were designed according to GEO, ENCODE and Roadmap Epigenomics database and were listed as the following: YAP Y1-Forward: 5'-TGT GCT TAG AAA GAG AGG GCT GCTG-3'; YAP Y1-Reverse: 5' -GAC GTG CAG TGA GTT CCA CAT CAA-3'; YAP Y2-Forward: 5'-CAG AGG AAG GAA GAG CCG AGA GG-3'; YAP Y2-Reverse: 5'-CGCCCGACTGAGACAGAAACT-3'; YAP Y3-Forward: 5'-GCG TGT TGG TTT CCC AGT TGT AGA-3'; YAP Y3-Reverse: 5'-GCG CAA CGT ACA GAT GTG GC TAAT-3'; YAP Y4-Forward: 5'-TGA CGA GGG CTT GAT TTT TCTTGA-3'; YAP Y4-Reverse: 5' -GTG CAG TTA TGG GTG CTT TTC CAA-3'.

\subsection{Luciferase reporter assay}

In order to study whether $\beta$-catenin could promote the transcription of Yap gene, we mutated the possible binding sites of $\beta$-catenin and Yap, TCF4 and Yap, according to the results of Chip. Luciferase reporter gene psicheck-2 (Control), psicheck-2-yap-wt, psicheck-2-yap-mut1 (TCF4), psicheck-2-yapmut2 ( $\beta$-catenin) were synthesized by Invitrogen (Shanghai, China). Psicheck-2-yap-wt referrers to Yap status wt. Psicheck-2-yap-mut1(TCF4) referrers to Yap mutated in possible binding sites of TCF4. Psicheck-2-yap-mut1( $\beta$-catenin) referrers to Yap mutated in possible binding sites of $\beta$-catenin. In order to further confirm whether $\beta$-catenin or TCF4 could promote Yap transcription. Over expression plasmid of $\beta$-catenin pcDNA3.1/EGFP/IRES- $\beta$-catenin was also synthesized by Invitrogen (Shanghai, 

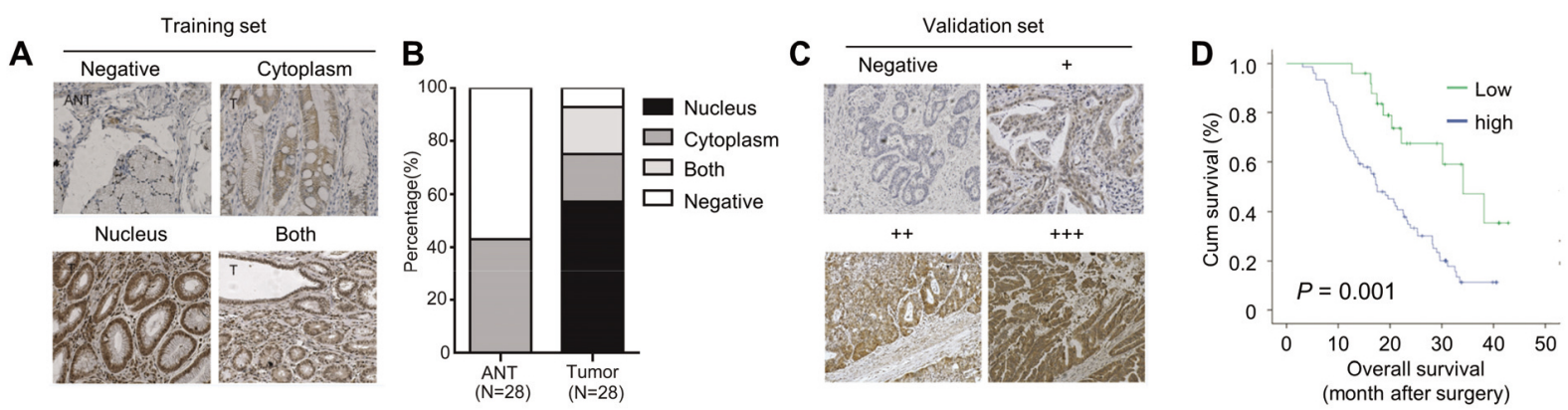

Fig. 1. YAP is upregulated in GC tissues and correlates with poor prognosis. x100. (A) Expressions of YAP in gastric cancer and corresponding adjacent non-tumor tissues detected by immunohistochemistry in training set. (B) Subcellular localization of YAP expression in 28 gastric cancer and corresponding adjacent non-tumor tissues. (C) Expressions of YAP in gastric cancer and corresponding adjacent non-tumor tissues detected by immunohistochemistry in validation set. (D) Kaplan-Meier analysis of overall survival time in 28 gastric cancer patients according to YAP expression.

China). Subsequently, we transfected synthetic plasmids and luciferase reporter gene into MGC-803 cells, including psicheck-2 (Control), psicheck-2-yap-wt, psicheck-2-yap-mut1 (TCF4), psicheck-2-yap-mut2 $(\beta$-catenin); and pcDNA3.1/EGFP/IRES- $\beta$-catenin + psicheck-2-yap-wt, pcDNA3.1/EGFP/IRES- $\beta$ catenin + psicheck-2-yap-mut ( $\beta$-catenin), psicheck-2-yap-wt+Vector. Dual Luciferase Reporter Assay System (Promega, Madison, WI, USA) was used to assess luciferase activity 48 hours later.

\subsection{Animal study}

The Institutional Animal Care and Use Committee of Nanjing Medical University (Nanjing, China) approved the animal study, which followed to the standards for the ethical review of laboratory animal welfare People's Republic of China National Standard GB/T 3589218. Four-Five weeks old BALB/c nude mice were utilized in this study. For tumor formation assay, a total of $1 \times 10^{6}$ stably knockdown YAP or negative control BGC823 cells were subcutaneously injected into each side of each mouse $(n=5)$. After that, we examined tumor growth and calculated tumor volumes every two days. For in vivo metastasis study, a total of $2 \times 10^{6}$ stably inhibiting YAP or control BGC823 cells were injected into the tail vein of 5 mice. Seven weeks following the injection, the mice were killed, their lungs were removed and imaged, and visible tumors on the lung surface were tallied. The growth of subcutaneous or lung metastatic tumors expressing GFP was monitored using the IVIS Spectrum In Vivo Imaging system (PerkinElmer, USA).

\subsection{Statistical analysis}

Statistical analysis was performed by SPSS 22.0 software (SPSS Inc., Chicago, IL). All of the data were presented as Means \pm SD. The clinical features of patients were assessed using chi-square test. The overall survival was analyzed by Kaplan-Meier method and log rank test. Cox proportional hazards model with $95 \%$ confidence interval (CI) was used for the univariate and multivariate analysis. All the experiments were repeated at least three times. The statistical significance of data was identified when $P$ value was less than 0.05 .

\section{Results}

\subsection{YAP is upregulated in GC tissues and correlates with poor prognosis}

The expressions of YAP in GC and corresponding adjacent non-tumor tissues (ANT) were detected by 
Table 1

Cox regression analysis of YAP expression and clinicpathologic variables in predicting OS of GC patients

\begin{tabular}{|c|c|c|c|c|c|c|}
\hline \multirow[t]{2}{*}{ Variables } & \multicolumn{3}{|c|}{ Univariate analysis } & \multicolumn{3}{|c|}{ Multivariate analysis } \\
\hline & HR & $95 \% \mathrm{CI}$ & $P$ & HR & $95 \% \mathrm{CI}$ & $P$ \\
\hline \multicolumn{7}{|l|}{ Age } \\
\hline$>60$ vs. $\leqslant 60$ & 0.81 & $0.51-1.30$ & 0.377 & 0.72 & $0.44-1.18$ & 0.191 \\
\hline \multicolumn{7}{|l|}{ Gender } \\
\hline Female vs. Male & 1.12 & $0.67-1.89$ & 0.655 & 1.42 & $0.80-2.54$ & 0.233 \\
\hline \multicolumn{7}{|l|}{ Histology } \\
\hline Non-adenocarcinoma vs. adenocarcinoma & 0.75 & $0.36-1.58$ & 0.453 & 0.68 & $0.31-1.46$ & 0.322 \\
\hline \multicolumn{7}{|l|}{ Differentiation } \\
\hline Poor Vs. well and moderate & 2.31 & $0.84-6.36$ & 0.106 & 1.49 & $0.50-4.43$ & 0.475 \\
\hline \multicolumn{7}{|l|}{ Tumor size } \\
\hline$\geqslant 6 \mathrm{Vs} .<6 \mathrm{~cm}$ & 0.94 & $0.57-1.55$ & 0.805 & 0.91 & $0.53-1.55$ & 0.732 \\
\hline \multicolumn{7}{|l|}{ TNM stage } \\
\hline IV Vs. III & 2.59 & $1.47-4.56$ & 0.001 & 4.13 & $2.14-7.95$ & $<0.001$ \\
\hline \multicolumn{7}{|l|}{ Vascular invasion } \\
\hline Yes Vs. no & 1.47 & $0.92-2.34$ & 0.108 & 0.93 & $0.49-1.77$ & 0.824 \\
\hline \multicolumn{7}{|l|}{ Nerval invasion } \\
\hline Yes Vs. no & 1.76 & $1.10-2.82$ & 0.018 & 1.84 & $0.94-3.60$ & 0.078 \\
\hline \multicolumn{7}{|l|}{ YAP } \\
\hline High Vs. low & 3.08 & $1.57-6.04$ & 0.001 & 3.58 & $1.74-7.37$ & 0.001 \\
\hline
\end{tabular}

IHC in our training set. We combined the fraction of positively stained tumor cells and the intensity of staining to calculate the fluorescence. Point 0 indicated negative, point $1-4$ indicated weak positive $(+)$, point 5-8 indicated medium positive (++) and point 9-13 indicated strong positive (+++). The cut off value of high-YAP and low-YAP was set as 4 . YAP was significantly upregulated in GC tissues compared with ANTs (Fig. 1A). In detail, YAP positive staining was found in 26 of $28 \mathrm{GC}$ samples and were demonstrated to be localized in cytoplasm (5 cases), nucleus (16 cases) and both (5 cases), respectively (Fig. 1A and B). Whereas, extremely weak levels of YAP were detected only in the cytoplasm of 13 ANTs (Fig. 1A and B). To verify the role of YAP in GC, we investigated the potential associations between YAP levels and GC patients' overall survival in our validation set. The patients were then divided into high-YAP and low-YAP groups according to their tumor YAP staining score (Fig. 1C and D). As shown in Fig. 1D, patients with high YAP expression in GC tissues had significantly worse prognosis than those with low YAP expression. Cox regression analysis of YAP expression and clinicpathologic variables in predicting OS of GC patients were shown in Table 1, and the results indicated that YAP might be an independent indicator for GC prognosis. Taken together, our results indicate that YAP is an oncogenic protein in GC.

\subsection{YAP promotes GC cells proliferation in vitro}

To investigate the biological functions of YAP in vitro, we then evaluated the endogenous level of YAP in GC cells lines by western blotting and qRT-PCR. As shown in Fig. 2A, YAP was highly expressed in SGC7901 and BGC823 cells. We designed YAP-shRNA1,YAP-shRNA2, YAP-shRNA3 and YAPshRNA4, which targeting different end of YAP transcript. By screening, we found that YAP-shRNA4 presented the highest knockdown efficiency of YAP in SGC7901 with inhibition rate of 55\%, while YAPshRNA2 possessed the vigorous inhibition efficiency in BGC823 with inhibition rate of 75\% (Fig. 2B and C). In the CCK8 assays, we found that downregulation of YAP lead to decrease in the proliferation ability of GC cells (Fig. 2D). Furthermore, YAP knockdown significantly suppressed clone formation ability in vitro (Fig. 2E). Taken together, our results indicate that YAP enhances the proliferation capacity in vitro. 

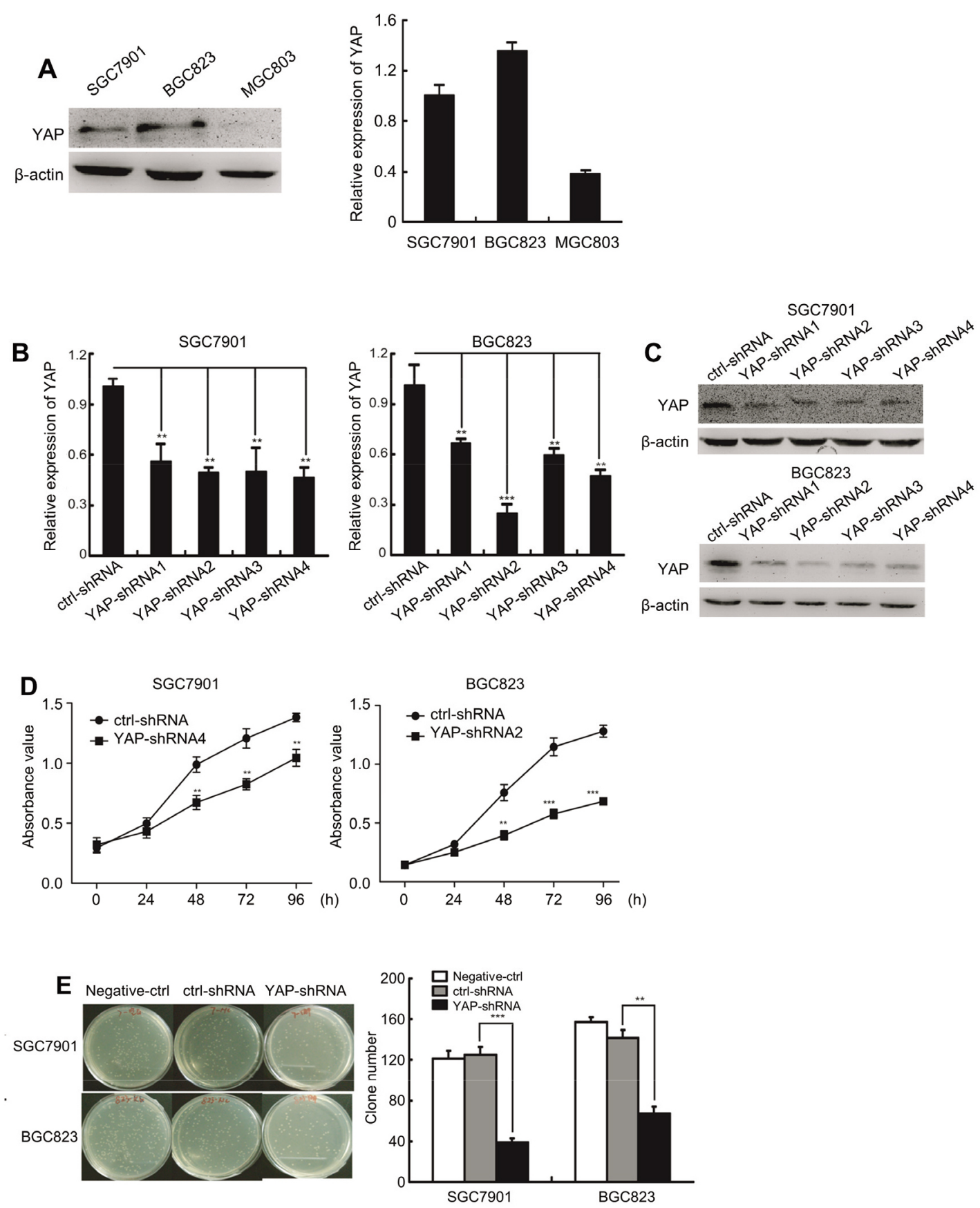

Fig. 2. YAP promotes GC cells proliferation in vitro. (A) The expression of YAP in SGC7901 and BGC823 cells. (B) The expression of YAP mRNA after transfected with YAP shRNA by RT-PCR in SGC7901 and BGC823 cells. (C) The expression of YAP protein after transfected with YAP shRNA by Western blot in SGC7901 and BGC823 cells. (D) Growth curves for control and YAP-shRNA silencing SGC7901 and BGC823 cells determined by CCK-8 assays. (E) Total number of colonies in SGC7901 and BGC823 cells transfected with negative control, shRNA control and YAP-shRNA by clonogenic assay. ${ }^{*} P<0.05,{ }^{* *} P<$ $0.01,{ }^{* * *} P<0.001$ 

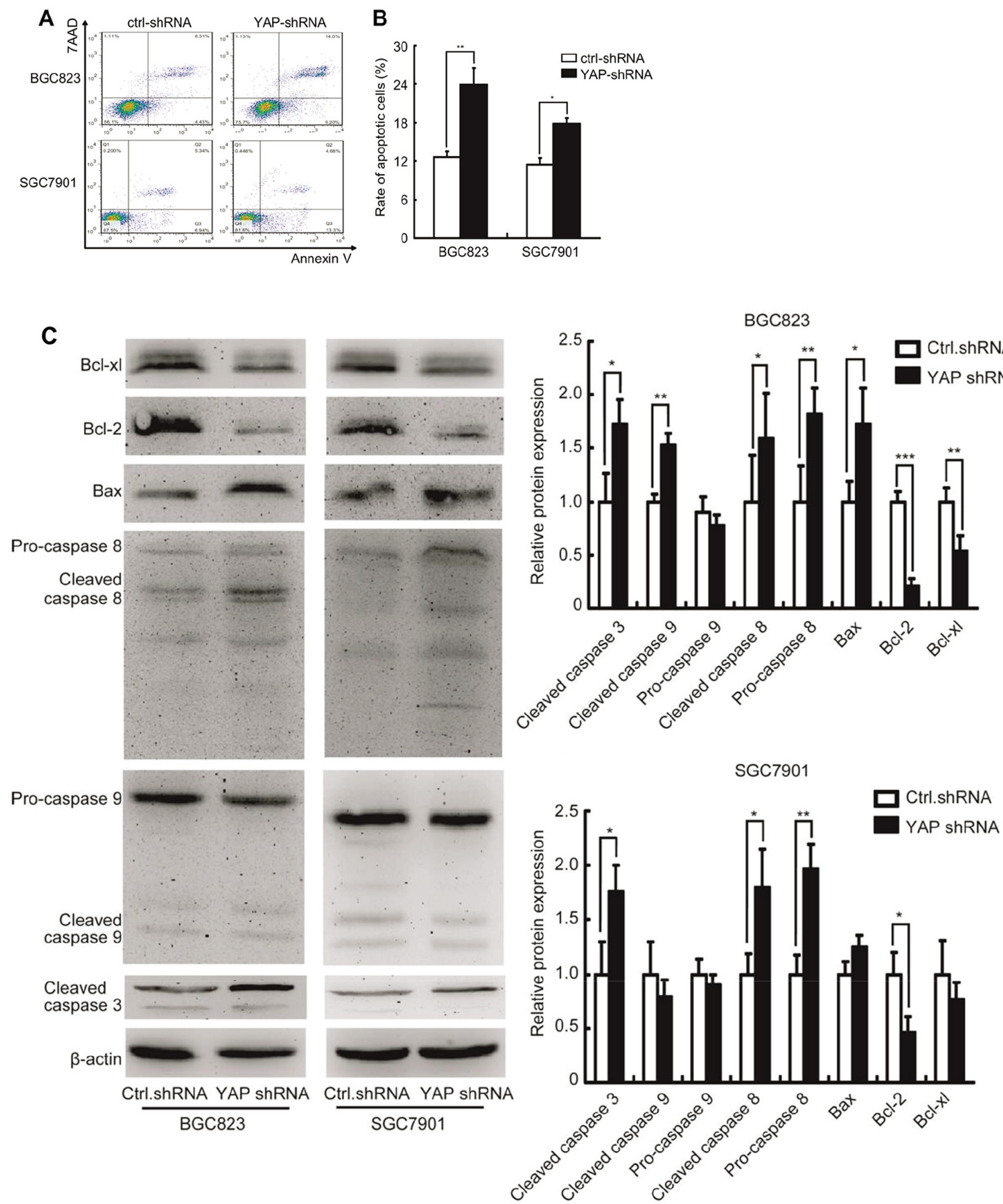

Fig. 3. Knockdown YAP1 promotes SGC7901 and BGC823 cells apoptosis in vitro. (A) YAP-shRNA induced cell apoptosis of SGC-7901 and BGC-823 cells detected by flow cytometry. (B) Rate of apoptosis cells of SGC-7901 and BGC-823 transfected with shRNA control and YAP-shRNA. (C) Expression of Bcl-xl, Bcl-2, Bax, pro-caspase8, cleaved-caspase8, pro-caspase9, cleaved-caspase9, cleaved-caspase 3 detected by Western blot. ${ }^{*} P<0.05,{ }^{* *} P<0.01,{ }^{* * *} P<0.001$. 

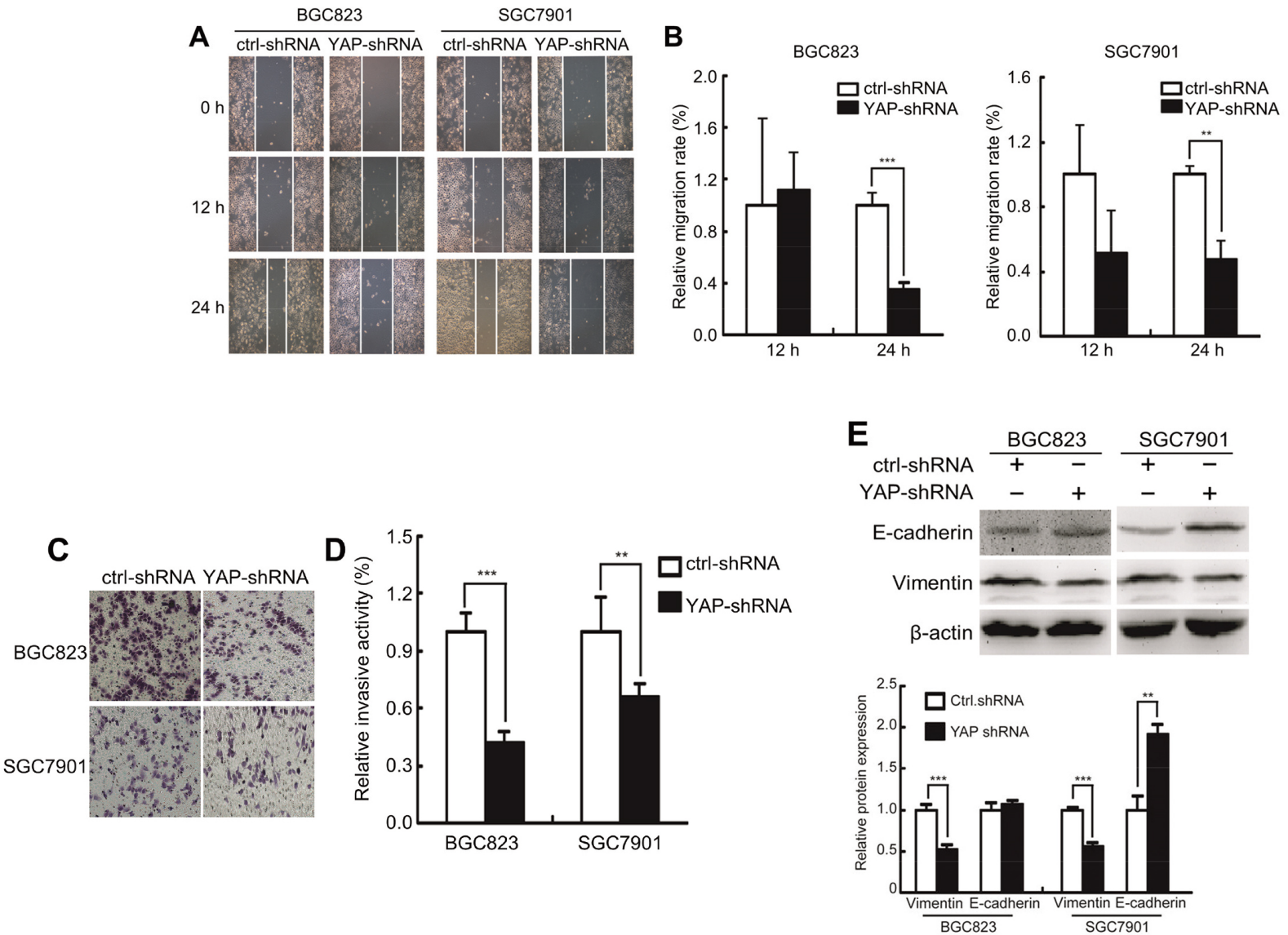

Fig. 4. YAP1 enhances the metastatic capacity of GC cells in vitro. (A) Migration capabilities of SGC7901 and BGC823 cells assessed by scratch wound assay. (B) Relative migration rate of SGC7901 and BGC823 cells assessed by scratch wound assay. (C) Invasion assay of SGC7901 and BGC823 cells transfected with shRNA control and YAP-shRNA. (D) Relative invasion activity of SGC7901 and BGC823 cells transfected with shRNA control and YAP-shRNA. (E) Expression of E-cadherin and vimentin of SGC7901 and BGC823 cells transfected with shRNA control and YAP-shRNA detected by Western blot. ${ }^{*} P<$ $0.05,{ }^{* *} P<0.01,{ }^{* * *} P<0.001$.

\subsection{YAP inhibits $G C$ cells apoptosis in vitro}

To investigate the role of YAP in GC cell apoptosis, the apoptotic cells were detected by FACS technology. As shown in Fig. 3A and B, suppression of YAP significantly promoted cell apoptosis in SGC7901 and BGC823. Furthermore, the abundance of cleaved-caspase8, cleaved-caspase3 and Bax were markedly increased upon YAP knockdown, while the levels of Bcl-xl and Bcl-2 were downregulated (Fig. 3C and D). Taken together, our results indicate that YAP knockdown promotes GC cells apoptosis in vitro.

\subsection{YAP1 enhances the metastatic capacity of GC cells in vitro}

To investigate whether YAP had a functional role in cell metastasis, the wound healing and transwell assays were conducted. As shown in Fig. 4A-D, the migration abilities of SGC7901 and BGC823 cells were significantly decreased when YAP was knockdown. Epithelial-mesenchymal transition (EMT) is 

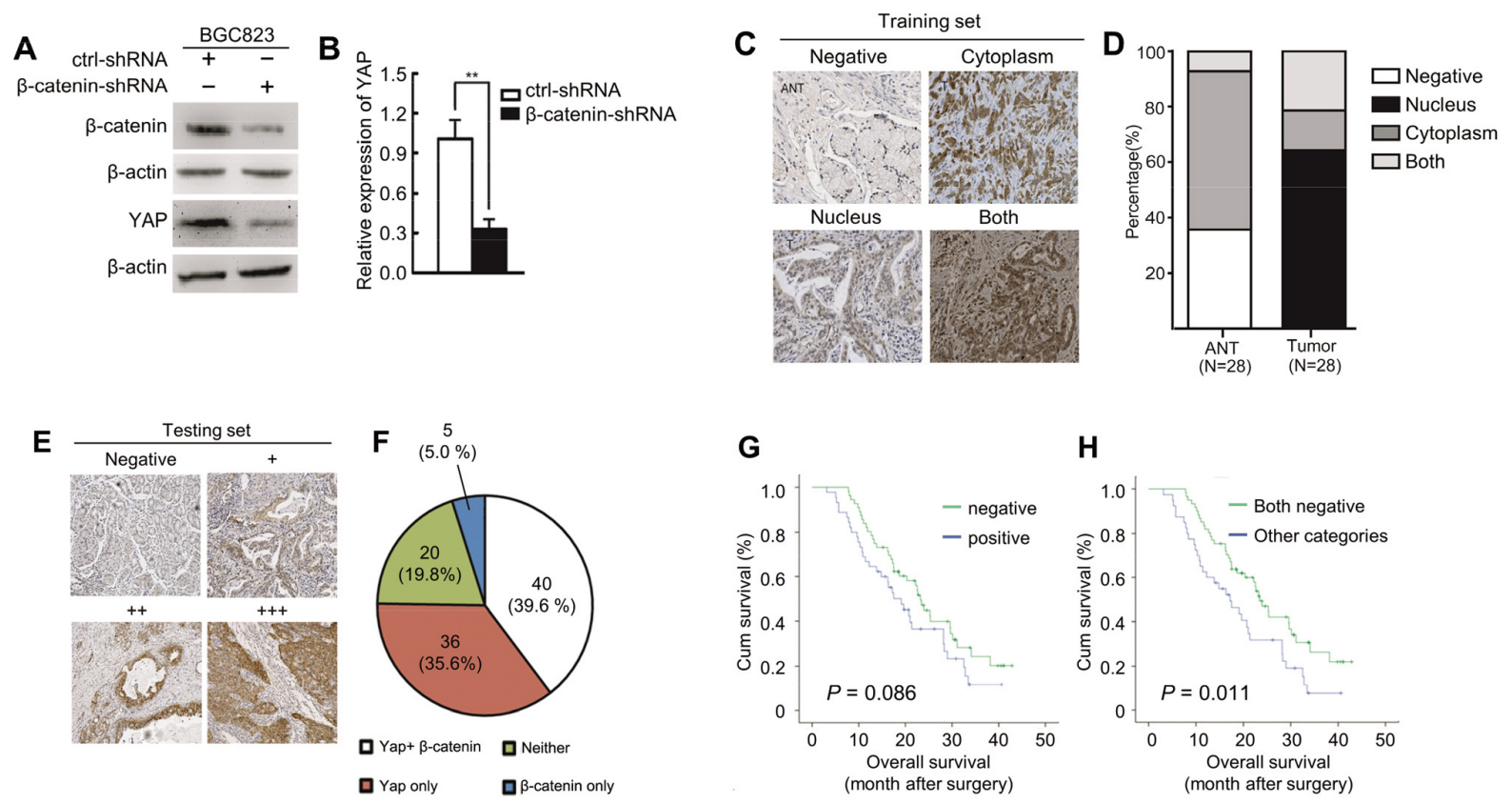

Fig. 5. $\beta$-catenin upregulates YAP expression and correlates with poor prognosis. (A) Protein level of YAP in BGC-823 cells transfected with shRNA control and $\beta$-catenin shRNA detected by Western blot. (B) Relative expression of YAP in BGC-823 cells transfected with shRNA control and $\beta$-catenin shRNA. (C) Expression of $\beta$-catenin in training set (28 gastric cancer samples and adjacent tissues) detected by immunohistochemistry. (D) Subcellular localization of $\beta$-catenin expression in training set (28 gastric cancer samples and adjacent tissues). (E) Expression of $\beta$-catenin in testing set (101 gastric cancer samples) detected by immunohistochemistry. (F) Correlation analysis for YAP and $\beta$-catenin by Spearman's correlation analysis. (G) Kaplan-Meier analysis of overall survival time in 101 gastric cancer patients according to $\beta$-catenin expression. $(\mathrm{H})$ Kaplan-Meier analysis of overall survival time in 101 gastric cancer patients according to both YAP and $\beta$-catenin expression. ${ }^{*} P<0.05,{ }^{* *} P<0.01$, ${ }^{* * *} P<0.001$.

essential for GC metastasis. In our study, we found that reducing YAP expressions substantially increased the E-cadherin (epithelial marker) levels, while decreased the vimentin (mesenchymal marker) levels (Fig. 4E). Taken together, our results indicate that YAP promotes the metastatic capacity of GC cells via EMT pathway.

\section{5. $\beta$-catenin upregulates YAP expression and correlates with poor prognosis}

To investigate the effect of $\beta$-catenin on the expression of YAP, we constructed a shRNA of $\beta$-catenin and then transfected it into BGC-823 cells. As shown in Fig. 5A and B, transfection with $\beta$-catenin shRNA led to decreased protein level of YAP. We further examined the expression of $\beta$-catenin in training set (28 gastric cancer samples and adjacent tissues as described previously) by immunohistochemistry. The IHC assay showed positive expression of $\beta$-catenin in all 28 gastric cancer samples. Among them, nuclear positive rate was $64.3 \%$ (18/28), cytoplasmic positive rate was $14.3 \%(4 / 28)$, the remaining $21.4 \%(6 / 28)$ samples showed both nuclear and cytoplasmic positive. In paired adjacent tissues, $\beta$-catenin wasn't expressed in $35.7 \%$ (10/28) samples and only 7.1\% (2/28) showed both nuclear and cytoplasmic positive (Fig. $5 \mathrm{C}$ and $\mathrm{D}$ ). We then examined the expression of $\beta$-catenin in testing set (101 GC samples as described previously). We combined the fraction of positively stained tumor cells and the intensity of staining to calculate the fluorescence. Point 0 indicated negative, point $1-4$ indicated weak positive 

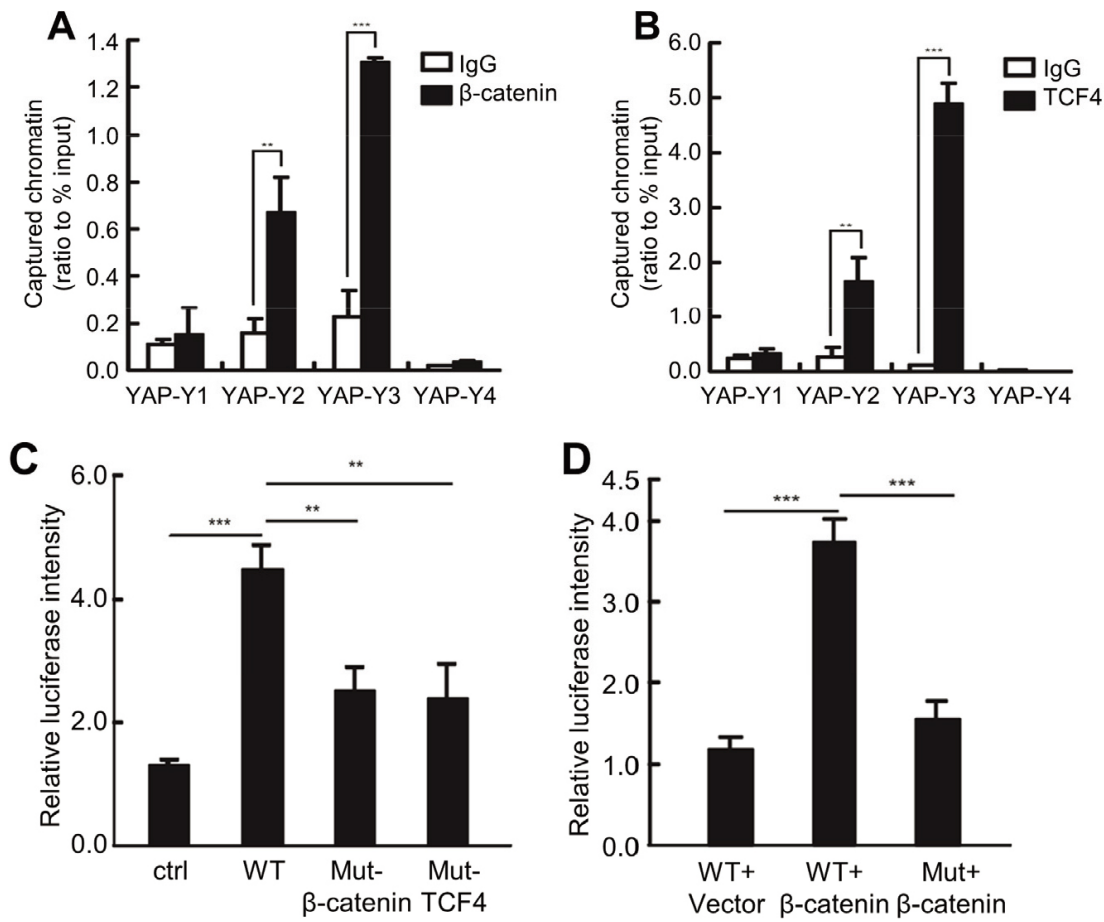

Fig. 6. $\beta$-catenin binds to the promotor region of YAP. (A) The relation of YAP and the promoter region $\beta$-catenin detected by CHIP assay. (B) The relation of YAP and the promoter region TCF4 detected by CHIP assay. (C) Luciferase reporter activity of BGC-823 cells transfected with psicheck-2 (Control), psicheck-2-yap-wt (WT), psicheck-2yap-mut1 (TCF4), psicheck-2-yap-mut2 ( $\beta$-catenin). (D) Luciferase reporter activity of BGC-823 cells transfected with pcDNA3.1/EGFP/IRES- $\beta$-catenin + psicheck-2-yap-wt, pcDNA3.1/EGFP/IRES- $\beta$-catenin + psicheck- 2 - yap-mut $(\beta$-catenin). ${ }^{*} P<0.05,{ }^{* *} P<0.01,{ }^{* * *} P<0.001$.

$(+)$, point 5-8 indicated medium positive $(++)$ and point $9-13$ indicated strong positive $(+++) . \beta$-catenin staining score more than 4 and located in the nucleus was considered as positive (Fig. 5E). Among them, nuclear positive rate was $44.6 \%$ (45/101). The Spearman's correlation analysis showed that, $39.6 \%$ (40/101) of 101 gastric cancer samples were nuclear positive for both $\beta$-catenin and YAP, 35.6\% (36/101) were nuclear positive for YAP only and 19.8\% (20/101) for nuclear $\beta$-catenin only (Fig. 5F). Spearman's correlation analysis showed positive relationship between YAP and $\beta$-catenin $(r=0.283, p=0.004)$. Survival analysis showed that $\beta$-catenin expression is correlated to a worse clinical outcome, but didn't reach statistical significance ( $p=0.086$, Fig. 5G). Interestingly, the overall survival of those with both YAP and $\beta$-catenin expression was significantly shorter than other groups ( $p=0.011$, Fig. $5 \mathrm{H})$.

\section{6. $\beta$-catenin binds to the promotor region of YAP}

We then performed the ChIP assay to demonstrate whether $\beta$-catenin binds to the promoter region of YAP directly. We designed 4 primers by consulting literature and software, and the sequence of primers was described in Materials and Methods. As we seen, $\beta$-catenin and TCF4 could directly bind to the promoter region of YAP-Y3 (Fig. 6A and B). Subsequently, luciferase reporter vectors containing psicheck-2 (Control), psicheck-2-yap-wt, psicheck-2-yap-mut1 (TCF4), psicheck-2-yap-mut2 ( $\beta$-catenin) were constructed and transfected into BGC-823 cells. Transfection of psicheck-2-yapmut1 (TCF4), psicheck-2-yap-mut2 ( $\beta$-catenin) significantly decreased the luciferase reporter activity 
A

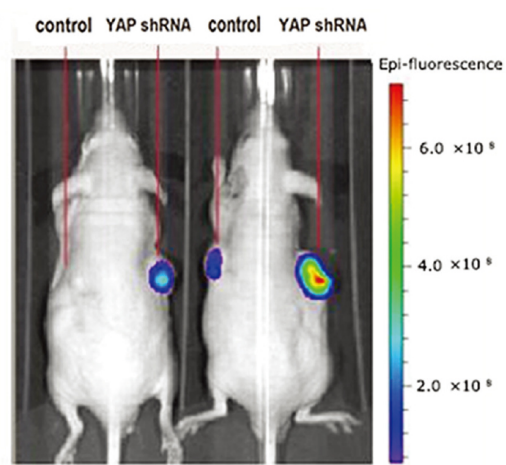

B

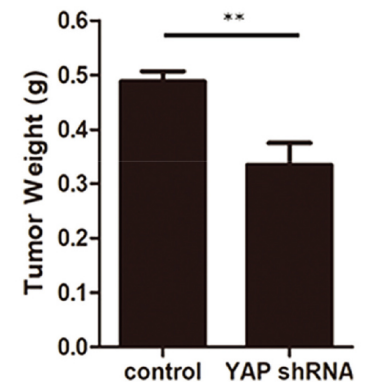

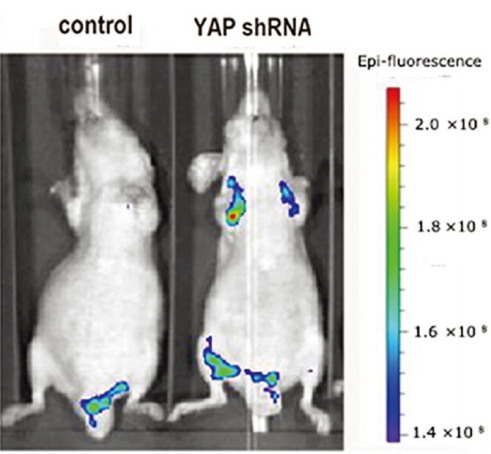

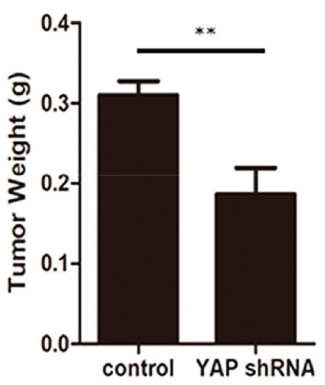

Fig. 7. YAP promotes GC cells proliferation and metastasis in vivo. (A) Analysis of tumor growth in vivo by injecting YAP stable knockdown BGC823 cells and control cells into nude mice. (B) Analysis of lung colonization by injecting YAP stable knockdown BGC823 cells and control cells into the tail vein of nude mice.

(Fig. 6C). Then we constructed plasmids containing pcDNA3.1/EGFP/IRES- $\beta$-catenin + psicheck-2-yapwt, pcDNA3.1/EGFP/IRES- $\beta$-catenin + psicheck-2-yap-mut ( $\beta$-catenin), psicheck-2-yap-wt+Vector and transfected them into BGC-823 cells. As we seen in Fig. 6D, plasmids containing yap-mut $+\beta$-catenin reduced luciferase reporter activity which was enhanced by yap-wt $+\beta$-catenin plasmids. Taken together, we found that $\beta$-catenin binded to a DNA promoter of the YAP gene.

\subsection{YAP promotes GC cells proliferation and metastasis in vivo}

We next used an athymic (nu/nu) animal model to investigate the role of YAP in proliferation induction. We injected YAP stable knockdown BGC823 cells or control cells into nude mice to see if YAP affected GC cell carcinogenesis in vivo. Tumors generated by stable knockdown cells of YAP were significantly smaller than those generated by control cells (Fig. 7A). We then investigated the role YAP played in lung colonization by tail veins injection. Mice injected with YAP stable knockdown BGC823 cells had a lower lung metastatic burden (Fig. 7B).

\section{Discussion}

Hippo pathway could regulate cell growth, division and death, making it a major target in cancer dysregulation. YAP is reported as an important component of Hippo signaling pathway [21,22]. Here we observed that YAP was significantly expressed in GC, especially in nuclear, which is consistent with previous studies [23-25]. The findings indicated that YAP might play a role in the development of gastric cancer. 
YAP may promote breast cancer cell growth by increasing the expression of cyclin D1 [26]. YAP signaling could also regulated autophagy and promoted cancer metastasis in triple negative breast cancer [27]. The aberrant activation of Hippo signaling pathway's downstream effectors YAP or TAZ has been identified as an attractive target in liver cancer [28]. Activation of LATS2/YAP pathway could also promote migration and invasion of non-small-cell lung cancer cell [29]. Here we found that YAP promotes gastric cells proliferation, inhibits gastric cells apoptosis and enhances the metastatic capacity of GC cells in vitro. We also found the YAP could promote GC cells proliferation and metastasis in vivo. Our results are consistent with previous literatures [30,31].

Ability to evade apoptosis has been recognized as a characteristic of tumorigenesis [32]. Mechanismly, we found that GC cells apoptosis by silencing YAP might be induced by the down-regulation of Bcl-xL, Bcl-2 and up-regulation of Bax and cleaved caspase 3, caspase 8. As we all know, caspase family is associated with cell death [33]. Caspase deficiency has been identified as a cause of tumour development [34]. The Bcl-2 family contains several essential apoptotic regulators. Among these, Bcl-xL and Bcl-2 prevent cells from entering apoptosis, whereas Bax induce cell death [33]. These results indicate that YAP inhibition affect GC cell proliferation and apoptosis at least partly via regulating the expression of Bcl-2, Bax and caspase.

Ability of invasion and metastasis has been recognized as another characteristic of tumorigenesis. EMT (epithelial to mesenchymal transition) event is now considered as an important factor associated with invasion and metastasis in cancer progression [35]. Epithelial cell could loosen adhesion structures, change their polarity, or rearrange their cytoskeleton [36]. In the process they expressed vimentin and downregulated E-cadherin [36]. Here we found that downregulated YAP expression could inhibit migration and invasion of GC cells and increased E-cadherin level and decreased vimentin level. These results indicate that YAP promotes GC cell metastatic capacity via EMT pathway.

Despite the fact that gene mutations in Hippo pathway seem to be rare, the activity of the Hippo pathway is clearly affected by crosstalk with signaling pathways [37]. Multiple cancer-associated signaling networks have been shown to interact with the Hippo pathway functionally, including the Notch, WNT, Hedgehog (HH), insulin, TGF $\beta-\mathrm{BMP}$ and mTOR pathways [13,37]. These signaling networks interact with the Hippo pathway in a regulatory manner, frequently at the level of the YAP onco-proteins [37]. Recently increasing number of studies indicated that many kinds of interactions exist between $\mathrm{Wnt} / \beta$ catenin and Hippo/YAP pathway [19,38-41]. YAP and TAZ have the potential to interact with $\beta$ catenin. They are essential components of the $\beta$-catenin degradation complex, which is responsible for orchestrating $\mathrm{Wnt}$ responses. They could inhibit $\mathrm{Wnt} / \beta$-catenin signaling by blocking $\beta$-catenin nuclear localization. It has been demonstrated that canonical $\mathrm{Wnt} / \beta$-catenin signaling plays a role in a variety of types of cancer [42-47]. In gastric cancer, FLangan et al. found that Wnt receptor Fzd7 played an essential role in gastric tumorigenesis [48]. YAP was also reported to enhance GC cell proliferation and migration by regulating Gli1 expression via the non-classical Hedgehog pathway [49]. We observed that $\beta$-catenin binds to the promotor region of YAP, upregulating YAP expression and correlates with poor prognosis. Our results are consistent with that of Wesley et al., who demonstrated that YAP expression in CRC cells is driven by $\beta$-catenin/TCF4 complexes, which bind to YAP DNA enhancer [19].

\section{Conclusion}

Taken together, we observed that YAP was elevated in GC tissues and was associated with worse prognosis. YAP could promote proliferation, inhibit apoptosis and enhance the metastatic capacity in GC cells and in vivo. We also found that both $\beta$-catenin and YAP in gastric cancer mostly expressed 
in nucleus. $\beta$-catenin could upregulate YAP expression by binding to the promotor region of YAP and correlates with poor prognosis. These findings suggest that therapeutics targeting the Hippo/YAP signaling pathway could be developed to treat patients with this disease.

\section{Acknowledgments}

This research was funded by the Advanced health talent of Six-One Project of Jiangsu province (grant number: LGY2017069).

\section{Conflict of interest}

The authors report no conflicts of interest related to this work.

\section{References}

[1] Siegel RL, Miller KD, Jemal A. Cancer statistics. CA Cancer J Clin. 2017; 67(1): 7-30. doi: 10.3322/caac.21387.

[2] Jeon J, Cheong JH. Clinical implementation of precision medicine in gastric cancer. J Gastric Cancer. 2019; 19(3): 235-253. doi: 10.5230/jgc.2019.19.e25.

[3] Zheng Y, Pan D. The hippo signaling pathway in development and disease. Dev Cell. 2019; 50(3): 264-282. doi: 10.1016/j.devcel.2019.06.003.

[4] Han Y. Analysis of the role of the Hippo pathway in cancer. J Transl Med. 2019; 17(1): 116. doi: 10.1186/s12967-019$1869-4$.

[5] Harvey KF, Pfleger CM, Hariharan IK. The Drosophila Mst ortholog, hippo, restricts growth and cell proliferation and promotes apoptosis. Cell. 2003; 114(4): 457-467.

[6] Hong W, Guan KL. The YAP and TAZ transcription co-activators: key downstream effectors of the mammalian Hippo pathway. Semin Cell Dev Biol. 2012; 23(7): 785-793. doi: 10.1016/j.semcdb.2012.05.004.

[7] Moroishi T, Hayashi T, Pan WW, Fujita Y, Holt MV, Qin J, et al. The hippo pathway kinases LATS1/2 suppress cancer immunity. Cell. 2016; 167(6): 1525-1539 e1517. doi: 10.1016/j.cell.2016.11.005.

[8] Huang J, Wu S, Barrera J, Matthews K, Pan D. The Hippo signaling pathway coordinately regulates cell proliferation and apoptosis by inactivating Yorkie, the Drosophila Homolog of YAP. Cell. 2005; 122(3): 421-434. doi: 10.1016/j.cell.2005.06.007.

[9] Yu FX, Zhao B, Guan KL. Hippo pathway in organ size control, tissue homeostasis, and cancer. Cell. 2015; 163(4): 811-828. doi: 10.1016/j.cell.2015.10.044.

[10] Cao L, Sun PL, Yao M, Jia M, Gao H. Expression of YES-associated protein (YAP) and its clinical significance in breast cancer tissues. Hum Pathol. 2017; 68: 166-174. doi: 10.1016/j.humpath.2017.08.032.

[11] Kim SH, Jin H, Meng RY, Kim DY, Liu YC, Chai OH, et al. Activating hippo pathway via Rassf1 by ursolic acid suppresses the tumorigenesis of gastric cancer. Int J Mol Sci. 2019; 20(19): doi: 10.3390/ijms20194709.

[12] Qu Y, Zhang L, Wang J, Chen P, Jia Y, Wang C, et al. Yes-associated protein (YAP) predicts poor prognosis and regulates progression of esophageal squamous cell cancer through epithelial-mesenchymal transition. Exp Ther Med. 2019; 18(4): 2993-3001. doi: 10.3892/etm.2019.7896.

[13] Irvine KD. Integration of intercellular signaling through the Hippo pathway. Semin Cell Dev Biol. 2012; 23(7): 812-817. doi: 10.1016/j.semcdb.2012.04.006.

[14] Fernandez LA, Northcott PA, Dalton J, Fraga C, Ellison D, Angers S, et al. YAP1 is amplified and up-regulated in hedgehog-associated medulloblastomas and mediates Sonic hedgehog-driven neural precursor proliferation. Genes Dev. 2009; 23(23): 2729-2741. doi: 10.1101/gad.1824509.

[15] Han H, Yang B, Nakaoka HJ, Yang J, Zhao Y, Le Nguyen K, et al. Hippo signaling dysfunction induces cancer cell addiction to YAP. Oncogene. 2018; 37(50): 6414-6424. doi: 10.1038/s41388-018-0419-5.

[16] Dierick H, Bejsovec A. Cellular mechanisms of wingless/Wnt signal transduction. Curr Top Dev Biol. 1999; 43: 153-190.

[17] Kimelman D, Xu W. beta-catenin destruction complex: insights and questions from a structural perspective. Oncogene. 2006; 25(57): 7482-7491. doi: 10.1038/sj.onc.1210055.

[18] Rosenbluh J, Nijhawan D, Cox AG, Li X, Neal JT, Schafer EJ, et al. beta-Catenin-driven cancers require a YAP1 transcriptional complex for survival and tumorigenesis. Cell. 2012; 151(7): 1457-1473. doi: 10.1016/j.cell.2012.11.026. 
[19] Konsavage WM, Jr., Kyler SL, Rennoll SA, Jin G, Yochum GS. Wnt/beta-catenin signaling regulates Yes-associated protein (YAP) gene expression in colorectal carcinoma cells. J Biol Chem. 2012; 287(15): 11730-11739. doi: 10.1074/jbc.M111.327767.

[20] Liu H, Liang S, Yang X, Ji Z, Zhao W, Ye X, et al. RNAi-mediated RPL34 knockdown suppresses the growth of human gastric cancer cells. Oncol Rep. 2015; 34(5): 2267-2272. doi: 10.3892/or.2015.4219.

[21] Steinhardt AA, Gayyed MF, Klein AP, Dong J, Maitra A, Pan D, et al. Expression of Yes-associated protein in common solid tumors. Hum Pathol. 2008; 39(11): 1582-1589. doi: 10.1016/j.humpath.2008.04.012.

[22] Zanconato F, Cordenonsi M, Piccolo S. YAP and TAZ: a signalling hub of the tumour microenvironment. Nat Rev Cancer. 2019; 19(8): 454-464. doi: 10.1038/s41568-019-0168-y.

[23] Zhang J, Yang YC, Zhu JS, Zhou Z, Chen WX. Clinicopathologic characteristics of YES-associated protein 1 overexpression and its relationship to tumor biomarkers in gastric cancer. Int J Immunopathol Pharmacol. 2012; 25(4): 977-987. doi: $10.1177 / 039463201202500415$.

[24] Song M, Cheong JH, Kim H, Noh SH, Kim H. Nuclear expression of Yes-associated protein 1 correlates with poor prognosis in intestinal type gastric cancer. Anticancer Res. 2012; 32(9): 3827-3834.

[25] Lu T, Sun L, Zhu X. Yes-associated protein enhances proliferation and attenuates sensitivity to cisplatin in human gastric cancer cells. Biomed Pharmacother. 2018; 105: 1269-1275. doi: 10.1016/j.biopha.2018.06.031.

[26] Wang X, Su L, Ou Q. Yes-associated protein promotes tumour development in luminal epithelial derived breast cancer. Eur J Cancer. 2012; 48(8): 1227-1234. doi: 10.1016/j.ejca.2011.10.001.

[27] Chen W, Bai Y, Patel C, Geng F. Autophagy promotes triple negative breast cancer metastasis via YAP nuclear localization. Biochem Biophys Res Commun. 2019; doi: 10.1016/j.bbrc.2019.09.133.

[28] Zhang S, Zhou D. Role of the transcriptional coactivators YAP/TAZ in liver cancer. Curr Opin Cell Biol. 2019; 61: 64-71. doi: 10.1016/j.ceb.2019.07.006.

[29] Wu T, Hu H, Zhang T, Jiang L, Li X, Liu S, et al. miR-25 promotes cell proliferation, migration, and invasion of non-small-cell lung cancer by targeting the LATS2/YAP signaling pathway. Oxid Med Cell Longev. 2019; 2019: 9719723. doi: 10.1155/2019/9719723.

[30] Sun D, Li X, He Y, Li W, Wang Y, Wang H, et al. YAP1 enhances cell proliferation, migration, and invasion of gastric cancer in vitro and in vivo. Oncotarget. 2016; 7(49): 81062-81076. doi: 10.18632/oncotarget.13188.

[31] Ajani JA, Xu Y, Huo L, Wang R, Li Y, Wang Y, et al. YAP1 mediates gastric adenocarcinoma peritoneal metastases that are attenuated by YAP1 inhibition. Gut. 2021; 70(1): 55-66. doi: 10.1136/gutjnl-2019-319748.

[32] Zeng Q, Hong W. The emerging role of the hippo pathway in cell contact inhibition, organ size control, and cancer development in mammals. Cancer Cell. 2008; 13(3): 188-192. doi: 10.1016/j.ccr.2008.02.011.

[33] Hengartner MO. The biochemistry of apoptosis. Nature. 2000; 407(6805): 770-776. doi: 10.1038/35037710.

[34] McIlwain DR, Berger T, Mak TW. Caspase functions in cell death and disease. Cold Spring Harb Perspect Biol. 2013; 5(4): a008656. doi: 10.1101/cshperspect.a008656.

[35] Chen T, You Y, Jiang H, Wang ZZ. Epithelial-mesenchymal transition (EMT): A biological process in the development, stem cell differentiation, and tumorigenesis. J Cell Physiol. 2017; 232(12): 3261-3272. doi: 10.1002/jcp.25797.

[36] Savagner P. The epithelial-mesenchymal transition (EMT) phenomenon. Ann Oncol. 2010; 21(Suppl 7): vii89-92. doi: 10.1093/annonc/mdq292.

[37] Harvey KF, Zhang X, Thomas DM. The Hippo pathway and human cancer. Nat Rev Cancer. 2013; 13(4): 246-257. doi: $10.1038 / \mathrm{nrc} 3458$

[38] Azzolin L, Panciera T, Soligo S, Enzo E, Bicciato S, Dupont S, et al. YAP/TAZ incorporation in the beta-catenin destruction complex orchestrates the Wnt response. Cell. 2014; 158(1): 157-170. doi: 10.1016/j.cell.2014.06.013.

[39] Deng F, Peng L, Li Z, Tan G, Liang E, Chen S, et al. YAP triggers the Wnt/beta-catenin signalling pathway and promotes enterocyte self-renewal, regeneration and tumorigenesis after DSS-induced injury. Cell Death Dis. 2018; 9(2): 153. doi: 10.1038/s41419-017-0244-8.

[40] Li N, Lu N, Xie C. The Hippo and Wnt signalling pathways: crosstalk during neoplastic progression in gastrointestinal tissue. FEBS J. 2019; 286(19): 3745-3756. doi: 10.1111/febs.15017.

[41] Kim M, Jho EH. Cross-talk between Wnt/beta-catenin and Hippo signaling pathways: a brief review. BMB Rep. 2014; 47(10): 540-545. doi: 10.5483/bmbrep.2014.47.10.177.

[42] Schneider JA, Logan SK. Revisiting the role of Wnt/beta-catenin signaling in prostate cancer. Mol Cell Endocrinol. 2018; 462(Pt A): 3-8. doi: 10.1016/j.mce.2017.02.008.

[43] MacDonald BT, Tamai K, He X. Wnt/beta-catenin signaling: components, mechanisms, and diseases. Dev Cell. 2009; 17(1): 9-26. doi: 10.1016/j.devcel.2009.06.016.

[44] Li X, Meng Y, Xie C, Zhu J, Wang X, Li Y, et al. Diallyl Trisulfide inhibits breast cancer stem cells via suppression of Wnt/beta-catenin pathway. J Cell Biochem. 2018; 119(5): 4134-4141. doi: 10.1002/jcb.26613.

[45] Krishnamurthy N, Kurzrock R. Targeting the Wnt/beta-catenin pathway in cancer: Update on effectors and inhibitors. Cancer Treat Rev. 2018; 62: 50-60. doi: 10.1016/j.ctrv.2017.11.002. 
[46] Arend RC, Londono-Joshi AI, Straughn JM, Jr., Buchsbaum DJ. The Wnt/beta-catenin pathway in ovarian cancer: a review. Gynecol Oncol. 2013; 131(3): 772-779. doi: 10.1016/j.ygyno.2013.09.034.

[47] Debebe A, Medina V, Chen CY, Mahajan IM, Jia C, Fu D, et al. Wnt/beta-catenin activation and macrophage induction during liver cancer development following steatosis. Oncogene. 2017; 36(43): 6020-6029. doi: 10.1038/onc.2017.207.

[48] Flanagan DJ, Barker N, Costanzo NSD, Mason EA, Gurney A, Meniel VS, et al. Frizzled-7 Is Required for Wnt Signaling in Gastric Tumors with and Without Apc Mutations. Cancer Res. 2019; 79(5): 970-981. doi: 10.1158/0008-5472.CAN18-2095.

[49] Han T, Cheng Z, Xu M, Wang X, Wu J, Fang X. Yes-associated protein contributes to cell proliferation and migration of gastric cancer via activation of Gli1. Onco Targets Ther. 2020; 13: 10867-10876. doi: 10.2147/OTT.S266449. 